\title{
Role of maternal immune activation in neurodevelopment disorders and possible pharmacological treatment
}

\author{
Aria Francesca ${ }^{1}$, Mastinu Andrea ${ }^{1}$, Maccarinelli Giuseppina ${ }^{1}$, Premoli Marika ${ }^{1}$, \\ Memo Maurizio $^{1}$, Bonini Sara Anna ${ }^{1}$. \\ 1 Department of Molecular and Translational Medicine, University of Brescia, \\ Brescia, Italy
}

Corrisponding author email: f.aria@unibs.it

\section{INTRODUCTION}

Aberrant immune activity during vulnerable and critical periods of neurodevelopment could participate in the generation of neurological dysfunction characteristic of several neurodevelopmental disorders (NDDs), including autism spectrum disorders and schizophrenia. Numerous epidemiological studies have shown a link between maternal infections and NDDs risk; animal models of Maternal Immune Activation (MIA) have confirmed this association. No current therapy exists for treatment or prevention of ASD. Drugs currently used for ASD are mainly symptomatic lacking specificity and are often ineffective for behavioural deficits. In order to find new drug therapy that acts on causes of ASD not on symptoms, a nonsteroidal anti-inflammatory drug, was used.

Materials and methods: WT dams was divided in three groups: dams treated with phosphate-buffered saline (PBS), dams treated with $0,3 \mathrm{mg} / \mathrm{kg}$ of lipopolysaccharide (LPS) subcutaneously (s.c.) alternate days and dams treated with LPS and $2 \mathrm{mg} / \mathrm{kg}$ of meloxicam per day throughout pregnancy. Adult (4-6 months) offspring of each group was analyzed in terms of inflammation, cortical structure and behavioral abnormalities.

Results: Adult offspring (LPS-mice) showed a significant increase in serum levels of interlukin-1 (IL-1 $\beta$ ) and significant increase in microglia (Iba1) and astrocytes (GFAP). Moreover LPS-mice display abnormal columnar organization in specific cortical areas and reduction in social interaction and an increase in repetitive behaviors. Interestingly, meloxicam reduced levels of IL- $\beta$ and GFAP but was not able to recover from alteration in cortical cytoarchitecture and behavioural deficits.

Discussion and conclusion: These data show that chronic exposure to inflammatory stimulus (LPS) during throughout pregnancy led to activation in maternal immune system that provoked aberrant change during neuronal foetal development. In addition, brain of this adult offspring showed cortical disorganization in specific cerebral areas involved in social and stereotype behaviours that are resulted change during behavioural tests. Preventive treatment with meloxicam reduced partially inflammation in offspring, but did not rescue from behavioural deficits, probably because during neuronal development are involved others proinflammatory factors not strictly related to cyclooxygenase activation.

\section{MELOXICAM}

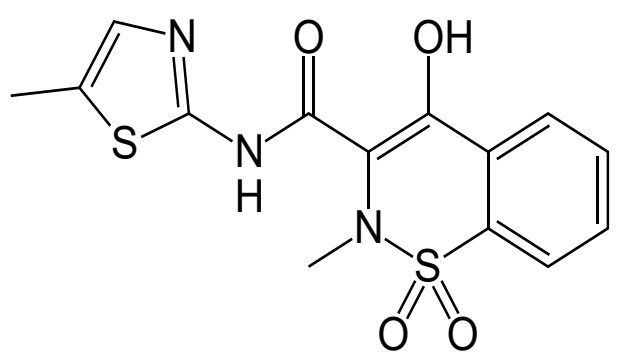

\section{PHARMACOLOGICAL TREATMENT}

WT dam was treated with $2 \mathrm{mg} / \mathrm{kg}$ Meloxicam per day by oral gavage throughout pregnancy.

Above is reported the chemical structure of Meloxicam. 


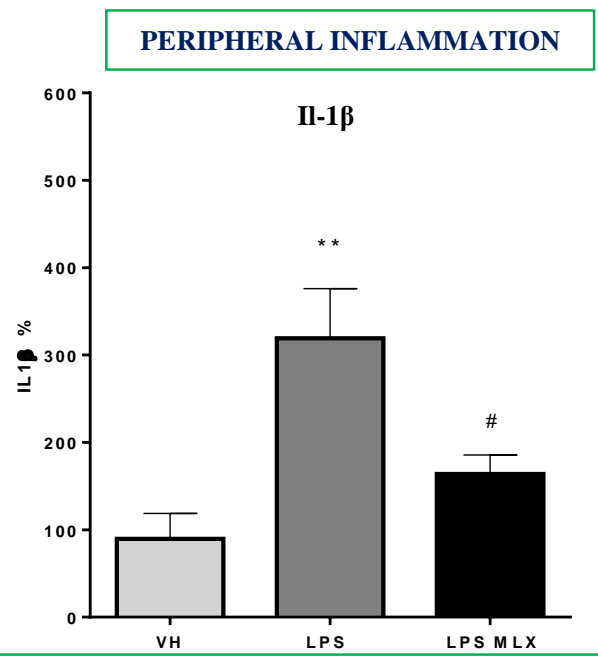

ELEVATED PERIPHERAL INFLAMMATION IN ADULT LPS-MICE

Levels of $1 \beta$ (IL-1 $\beta$ ) in VH, LPS, LPS+MLX offspring were measured by ELISA. ** $(\mathrm{p}<0,005)$ for LPS vs VH by One-way ANOVA followed by the Tukey post-test analysis.

\# $(\mathrm{p}<0,05)$ for LPS+MLX vs LPS by one-way ANOVA followed by the Tukey post-test analysis.

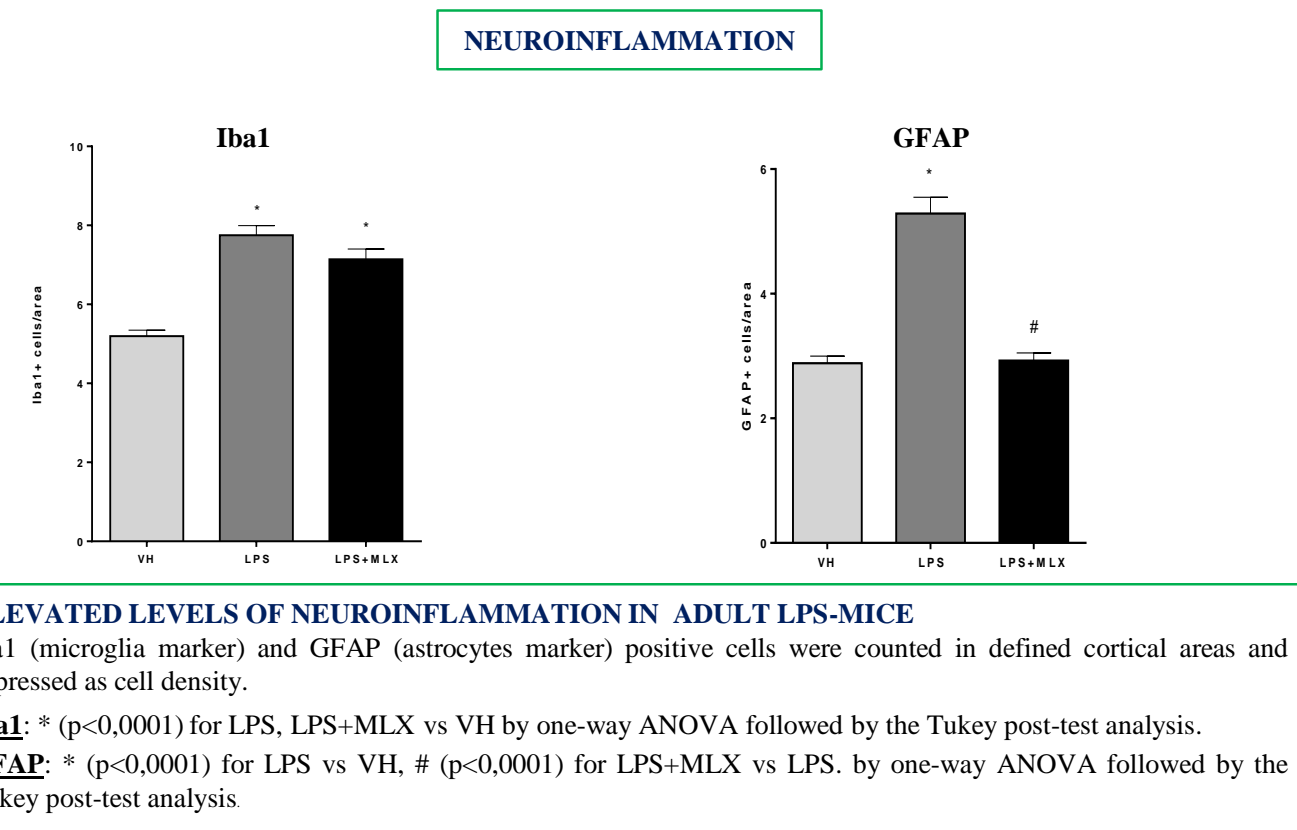

\section{CORTICAL CYTOARCHITECTURE}
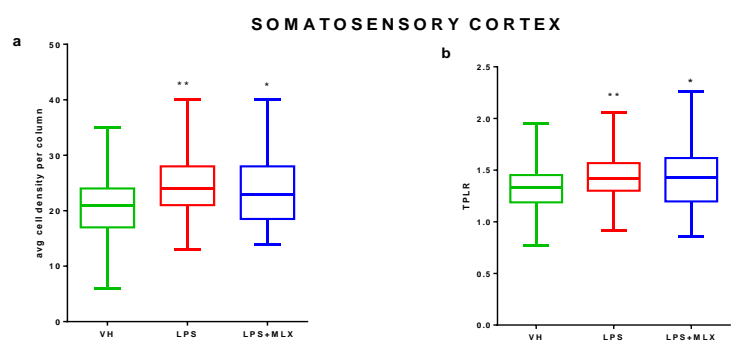

\section{ABNORMAL COLUMNAR ORGANIZATION} IN ADULT LPS-MICE CORTEX

Graphic representation of data obtained from the minicolumns analysis in VH, LPS and LPS+MLX adult offspring cortex. The following parameters were measured and reported in the graph: the average cell density per column and total path lenght ratio (TPLR).

CINGULATE CORTEX

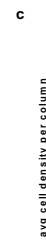
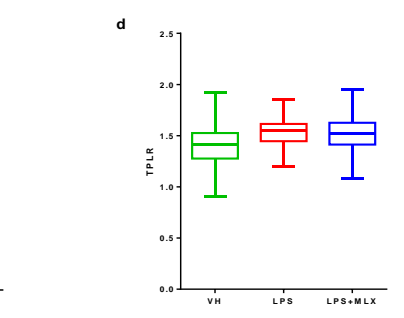

Somatosensory Cortex: $*(\mathrm{p}<0,0001)$ for LPS vs $\mathrm{VH}, *(\mathrm{p}<0,0005)$ for LPS+MLX vs VH by oneway ANOVA followed by the Tukey post-test analysis.

Cingulate Cortex: * $(\mathrm{p}<0,0001)$ for LPS vs VH by one-way ANOVA followed by the Tukey post-test analysis. 


\section{OPEN FIELD}
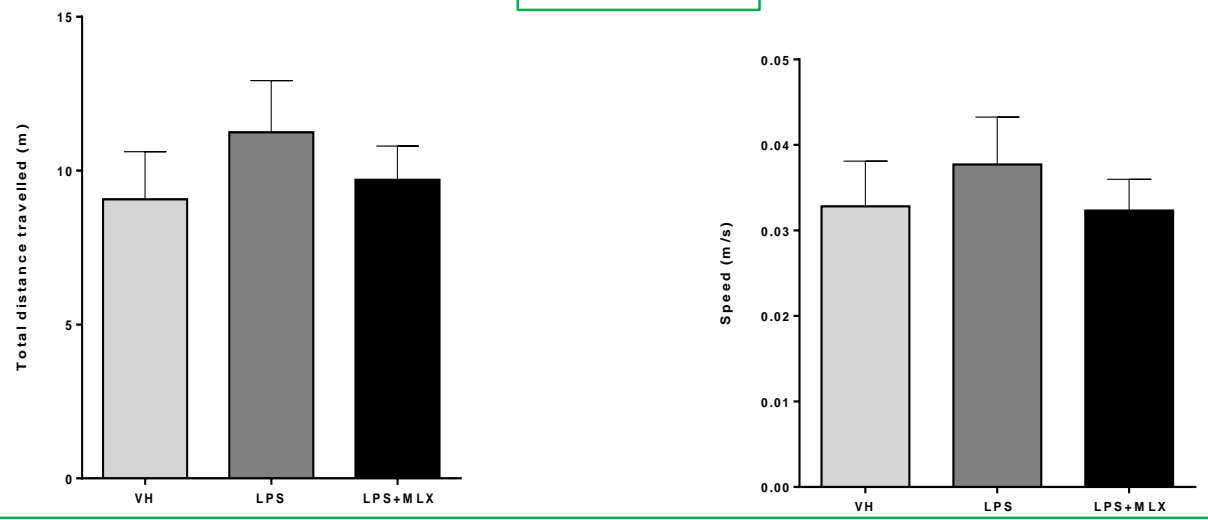

\section{NO DIFFERENCE IN EXPLORATORY ACTIVITY IN ADULT LPS-MICE}

Graphic representation of data collected in the 5 minutes open field test from VH, LPS, LPS+MLX adult offspring by automatically measuring the total distance travelled and the average speed.

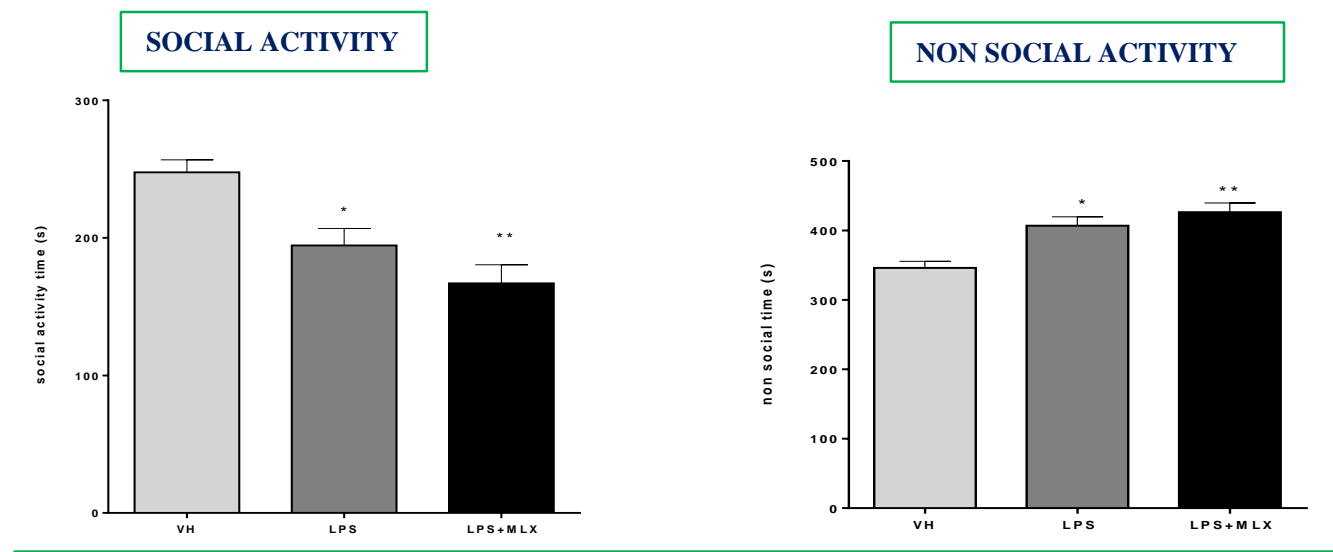

\section{DEFICIT IN SOCIAL INTERACTION IN ADULT LPS-MICE}

Graphic representation of data obtained from social behavioural analysis of VH, LPS and LPS+MLX adult offspring. Social activities include: sniffing, wrestling, following, mounting and pushing. Non social activities include: exploring, self-grooming and climbing.

* $(\mathrm{p}<0.05)$ for LPS vs VH, ** ( $<$ < 0.0001) for LPS+MLX vs VH by one-way ANOVA followed by the Sidak's multiple comparison test.

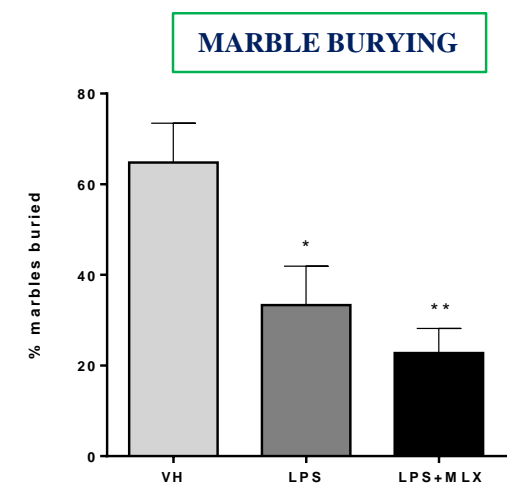

REDUCED BURYING ACTIVITY IN ADULT LPS-MICE

Graphic representation of data obtained from the number of marbles buried by VH, LPS and LPS+MLX adult offspring.

$*$ (p $<0.05)$ for LPS vs VH and ** (p< $0.0005)$ for LPS+MLX vs VH by one-way ANOVA followed by the Sidak's multiple comparison test.

\section{STEREOTYPED BEHAVIOR}

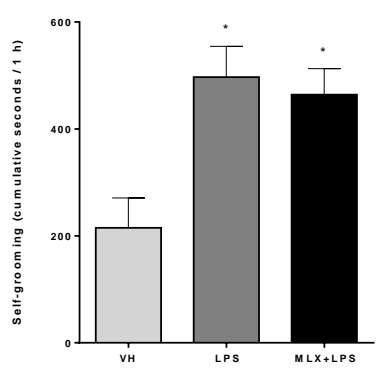

INCREASED REPETITIVE BEHAVIOURS IN ADULT LPS-MICE

Graphic representation of data obtained from spontaneous activity analysis of VH, LPS and LPS+MLX adult offspring.

$*(\mathrm{p}<0.01)$ for LPS and LPS+MLX vs VH by one-way ANOVA followed by the Sidak's multiple comparison test. 